\title{
ROTHE METHOD FOR A MIXED PROBLEM WITH AN INTEGRAL CONDITION FOR THE TWO-DIMENSIONAL DIFFUSION EQUATION
}

\author{
NABIL MERAZGA AND ABDELFATAH BOUZIANI \\ Received 8 April 2003
}

This paper deals with an initial boundary value problem with an integral condition for the two-dimensional diffusion equation. Thanks to an appropriate transformation, the study of the given problem is reduced to that of a onedimensional problem. Existence, uniqueness, and continuous dependence upon data of a weak solution of this latter are proved by means of the Rothe method. Besides, convergence and an error estimate for a semidiscrete approximation are obtained.

\section{Introduction}

Let $\Omega \subset \mathbb{R}^{2}$ be the open unit square $(0,1) \times(0,1)$ and $I$ the time interval $[0, T]$. The purpose of this paper is to study the solvability of the following two-dimensional equation:

$$
\frac{\partial \theta}{\partial t}-\left(\frac{\partial^{2} \theta}{\partial x^{2}}+\frac{\partial^{2} \theta}{\partial y^{2}}\right)=\varphi(x, y, t), \quad(x, y, t) \in \Omega \times I,
$$

with the initial condition

$$
\theta(x, y, 0)=\theta_{0}(x, y), \quad(x, y) \in \Omega,
$$

the Neumann conditions

$$
\begin{aligned}
& \frac{\partial \theta}{\partial x}(0, y, t)=\mu_{0}(y, t), \quad(y, t) \in(0,1) \times I, \\
& \frac{\partial \theta}{\partial x}(1, y, t)=\mu_{1}(y, t) p(t), \quad(y, t) \in(0,1) \times I, \\
& \frac{\partial \theta}{\partial y}(x, 0, t)=\eta_{0}(x, t), \quad(x, t) \in(0,1) \times I, \\
& \frac{\partial \theta}{\partial y}(x, 1, t)=\eta_{1}(x, t), \quad(x, t) \in(0,1) \times I,
\end{aligned}
$$

Copyright (C) 2003 Hindawi Publishing Corporation

Abstract and Applied Analysis 2003:16 (2003) 899-922

2000 Mathematics Subject Classification: 35K05, 35K20, 35B30, 35B45, 35D05

URL: http://dx.doi.org/10.1155/S1085337503305019 
and the integral condition

$$
\int_{0}^{1} \int_{0}^{1} \theta(x, y, t) d x d y=E(t), \quad t \in I,
$$

where $\varphi, \theta_{0}, \mu_{0}, \mu_{1}, \eta_{0}, \eta_{1}$, and $E$ are given functions supposed to be sufficiently regular, while the functions $\theta$ and $p$ are unknown. Here, the time-dependent parameter $p$ is a control function.

The diffusion equation with an integral condition can model various physical phenomena in the context of chemical engineering, thermoelasticity, population dynamics, heat conduction processes, control theory, medical science, life sciences, and so forth (see $[5,13]$ and the references therein). It is the reason for which such problems gained much attention in recent years, not only in engineering but also in the mathematics community.

Most of the papers dealing with problems of this type were consecrated to one-dimensional equations. The first work in this direction goes back to Cannon [6]. The author, with the aid of an equivalent integral equation, proved the existence and uniqueness of the classical solution for a mixed problem with an integral condition for the homogeneous one-dimensional heat equation.

In different approaches, mixed problems for second-order one-dimensional parabolic equations which combine Dirichlet and integral conditions were investigated by Kamynin [18], Ionkin [15], Cannon and van der Hoek [9, 10], Yurchuk [27], Benouar and Yurchuk [1], and Bouziani [5]. With regard to mixed problems for one-dimensional heat equation with Neumann and integral conditions, Cannon et al. [8] and Cannon and Hoek [11] presented numerical schemes based on finite difference method, Shi [26] established the well-posedness of the problem in a weighted fractional Sobolev space by means of the Fourier transform and a variational formulation. For similar problems for more general parabolic equations, we refer the reader to $[2,3,4]$ in which the author used the energy-integral method to study the solvability of the posed problems in a strong sense.

As for two-dimensional homogeneous diffusion equations with an integral condition, they have recently been treated in $[7,12,13,14]$.

Unlike all previous works, here we will prove the solvability of problem (1.1)(1.4) via approximation by the Rothe time-discretization method (also called method of lines) after reduction to a one-space variable problem. This method is a convenient tool for both the theoretical and the numerical analysis of the studied problem. Indeed, in addition to giving the first step towards a fully discrete approximation scheme, it provides a constructive proof of the existence of a unique exact solution to the investigated problem.

We note that since 1930, the Rothe method has been used several times to solve a relatively broad complex of evolution problems by many authors (cf., e.g., $[16,17,19,20,21,22,23,24])$. However, up to now, no evolution problem with an integral condition over the spatial domain has been treated with the help of 
this method. So, our paper can be considered as a contribution to the extension of the field of application of the aforesaid method to a new kind of problems.

The paper is organized as follows. In Section 2, we show that the investigation of problem (1.1)-(1.4) can be reduced to that of the one-dimensional problem (2.2)-(2.5) via a suitable transformation. We also give notation and assumptions on data. In Section 3, we solve the time-discretized problems corresponding to (2.2)-(2.5). Then, we derive some a priori estimates for the approximations and establish convergence and existence result for problem (2.2)-(2.5) by considering firstly the case of homogeneous boundary conditions in Section 4 and secondly the nonhomogeneous case in Section 5.

\section{Preliminaries}

Exploiting an idea due to Dehghan [13], we reformulate problem (1.1)-(1.4) by introducing a new unknown function $u:(0,1) \times I \rightarrow \mathbb{R}$ defined as follows:

$$
u(x, t)=\int_{0}^{1} \theta(x, y, t) d y
$$

Then, we have to find a function $u(x, t)$ such that

$$
\begin{aligned}
\frac{\partial u}{\partial t}-\frac{\partial^{2} u}{\partial x^{2}} & =f(x, t), \quad(x, t) \in(0,1) \times I, \\
u(x, 0) & =U_{0}(x), \quad x \in(0,1), \\
\frac{\partial u}{\partial x}(0, t) & =\alpha(t), \quad t \in I, \\
\int_{0}^{1} u(x, t) d x & =E(t), \quad t \in I
\end{aligned}
$$

where

$$
\begin{aligned}
f(x, t) & =\int_{0}^{1} \varphi(x, y, t) d y+\eta_{1}(x, t)-\eta_{0}(x, t), \\
U_{0}(x) & =\int_{0}^{1} \theta_{0}(x, y) d y, \\
\alpha(t) & =\int_{0}^{1} \mu_{0}(y, t) d y .
\end{aligned}
$$

Hence, once the solution of problem (2.2)-(2.5) is obtained, the value of $p$ will be obtained through the following formula:

$$
p(t)=\frac{(\partial u / \partial x)(1, t)}{\int_{0}^{1} \mu_{1}(y, t) d y}
$$


902 On the diffusion equation with an integral condition

provided that $\int_{0}^{1} \mu_{1}(y, t) d y \neq 0$ and $u$ is smooth enough so that $(\partial u / \partial x)(1, t)$ makes sense. Afterwards, (2.7) will be used to find $\theta$ as the solution of problem (1.1)-(1.3) with classical boundary conditions of Neumann type, whose investigation is standard numerically as well as analytically. Thus, the study of problem (1.1)-(1.4) is simply reduced to that of problem (2.2)-(2.5). We then concentrate on this latter.

In the course of this paper, $(\cdot, \cdot)$ denotes the usual scalar product in $L^{2}(0,1)$ and $\|\cdot\|$ the corresponding norm. We denote by $V$ the set of all $\phi \in L^{2}(0,1)$ which fulfil $\int_{0}^{1} \phi(x) d x=0$. Obviously, $V$ is a closed linear subspace of $L^{2}(0,1)$ and, consequently, it is a Hilbert space for the $L^{2}(0,1)$-inner product. By $H^{2}(0,1)$ we denote the usual second-order Sobolev space on $(0,1)$ with norm $\|\cdot\|_{H^{2}(0,1)}$. Let $X$ be a normed linear space. Then $L^{2}(I, X)$ and $L^{\infty}(I, X)$ denote the sets of all measurable functions $v: I \rightarrow X$ such that

$$
\begin{gathered}
\|v\|_{L^{2}(I, X)}^{2}=\int_{I}\|v(t)\|_{X}^{2} d t<\infty, \\
\|v\|_{L^{\infty}(I, X)}=\underset{t \in I}{\operatorname{ess} \sup }\|v(t)\|_{X}<\infty,
\end{gathered}
$$

respectively. By $C(I, X)$ and $C^{0,1}(I, X)$ we denote the sets of continuous and Lipschitz continuous mapping $v: I \rightarrow X$, respectively, where the first one is normed by

$$
\|v\|_{C(I, X)}=\max _{t \in I}\|v(t)\|_{X}
$$

while by $C^{1,1}(I, X)$ we denote the subset of all $v \in C^{0,1}(I, X)$ such that $d v / d t$ $\in C^{0,1}(I, X)$.

Moreover, our analysis requires the use of the nonclassical function space $B_{2}^{1}(0,1)$ introduced by Bouziani in [4] in the following way.

Let $C_{0}(0,1)$ be the space of real continuous functions with compact support in $(0,1)$. Since such functions are Lebesgue-integrable, we can define on $C_{0}(0,1)$ the following inner product:

$$
(u, v)_{B}=\int_{0}^{1} \mathfrak{J}_{x} u \cdot \mathfrak{J}_{x} v d x
$$

where $\mathfrak{J}_{x} v=\int_{0}^{x} v(\xi) d \xi$ for every fixed $x \in(0,1)$. If $\|\cdot\|_{B}$ denotes the corresponding norm, that is,

$$
\|v\|_{B}=\sqrt{(v, v)_{B}}=\left\|\mathfrak{I}_{x} v\right\|
$$

the inequality

$$
\|v\|_{B}^{2} \leqslant \frac{1}{2}\|v\|^{2}
$$


holds for every $v \in C_{0}(0,1)$. This fact implies that $C_{0}(0,1)$ is not complete for $(\cdot, \cdot)_{B}$, otherwise it would be so too for $(\cdot, \cdot)$, which is not true. Denote by $B_{2}^{1}(0,1)$ the completion of $C_{0}(0,1)$ for this new inner product. Then, from $(2.12)$, we readily see that $L^{2}(0,1)$ is a subset of $B_{2}^{1}(0,1)$ and, furthermore, the embed$\operatorname{ding} L^{2}(0,1) \rightarrow B_{2}^{1}(0,1)$ is continuous. Note that, by a density argument, inequality $(2.12)$ can be extended to functions in $L^{2}(0,1)$.

In the sequel, any function $(x, t) \in(0,1) \times I \mapsto g(x, t) \in \mathbb{R}$ is automatically identified with the associated abstract function $t \mapsto g(t)$ defined from $I$ into some function space on $(0,1)$ by setting $g(t): x \in(0,1) \mapsto g(x, t)$. The strong convergence is denoted by $\rightarrow$, while $\rightarrow$ and $\stackrel{*}{\rightarrow}$ stand for the weak and weak* convergence, respectively. By $C$ we denote a generic positive constant.

We formulate the following assumptions which are supposed to hold throughout the paper:

$\left(\mathrm{A}_{1}\right) f(t) \in L^{2}(0,1)$ for each $t \in I$, and the Lipschitz condition

$$
\left\|f(t)-f\left(t^{\prime}\right)\right\|_{B_{2}^{1}(0,1)} \leqslant l\left|t-t^{\prime}\right|
$$

holds for arbitrary $t, t^{\prime} \in I$;

$\left(\mathrm{A}_{2}\right) \alpha, E \in C^{1,1}(I, \mathbb{R})$;

$\left(\mathrm{A}_{3}\right) U_{0} \in H^{2}(0,1)$;

$\left(\mathrm{A}_{4}\right)$ the compatibility conditions are $\left(d U_{0} / d x\right)(0)=\alpha(0)$ and $\int_{0}^{1} U_{0}(x) d x=$ $E(0)$.

We look for a weak solution in the following sense.

Definition 2.1. A function $u: I \rightarrow L^{2}(0,1)$ is called a weak solution to problem (2.2)-(2.5) if the following conditions are satisfied:

(i) $u \in L^{2}\left(I, L^{2}(0,1)\right) \cap C\left(I, B_{2}^{1}(0,1)\right)$;

(ii) $u$ has a strong derivative $d u / d t \in L^{2}\left(I, B_{2}^{1}(0,1)\right)$;

(iii) $u$ fulfils the initial condition (2.3) and the integral condition (2.5);

(iv) let $\gamma:(0,1) \times I \rightarrow \mathbb{R}$ be the function

$$
\gamma(x, t)=\alpha(t)\left(x-\frac{1}{2}\right)+E(t), \quad x \in(0,1), t \in I .
$$

Then the integral identity

$$
\begin{aligned}
\int_{I}\left(\frac{d u}{d t}(t),\right. & v(t))_{B_{2}^{1}(0,1)} d t+\int_{I}(u(t), v(t)) d t \\
& =\int_{I}(f(t), v(t))_{B_{2}^{1}(0,1)} d t+\int_{I}(\gamma(t), v(t)) d t
\end{aligned}
$$

holds for all $v \in L^{2}(I, V)$.

We remark that due to (i), condition (iii) has sense, and by virtue of (i), (ii), and assumption $\left(\mathrm{A}_{2}\right)$, each term in the integral relation (iv) is well defined. 
904 On the diffusion equation with an integral condition

\section{Solvability of time-discretized problems}

In order to solve problem (2.2)-(2.5) by the Rothe method, we subdivide the time interval $I$ by points $t_{j}=j h, j=0, \ldots, n$, where $h=T / n$ is a step time. Then, for each $n \geqslant 1$, problem (2.2)-(2.5) may be approximated by the following recurrent sequence of time-discretized problems.

Starting from

$$
u_{0}=U_{0}
$$

find, successively for $j=1, \ldots, n$, functions $u_{j}:(0,1) \rightarrow \mathbb{R}$ such that

$$
\begin{gathered}
\delta u_{j}-u_{j}^{\prime \prime}=f_{j}, \quad x \in(0,1), \\
u_{j}^{\prime}(0)=\alpha_{j}, \\
\int_{0}^{1} u_{j}(x) d x=E_{j},
\end{gathered}
$$

where $\delta u_{j}=\left(u_{j}-u_{j-1}\right) / h, f_{j}=f\left(x, t_{j}\right), \alpha_{j}=\alpha\left(t_{j}\right), E_{j}=E\left(t_{j}\right)$, and ' or " stands for the first or the second derivative with respect to $x$, respectively.

Because of the nonclassical condition (3.4), no standard method can be directly used to solve (3.2)-(3.4). Following an idea of [25], we consider the auxiliary Neumann boundary value problem for a second-order linear ordinary differential equation

$$
\begin{gathered}
-w_{j}^{\prime \prime}+\frac{1}{h} w_{j}=f_{j}+\frac{1}{h} w_{j-1}, \quad x \in(0,1), \\
w_{j}^{\prime}(0)=\alpha_{j}, \\
w_{j}^{\prime}(1)=\lambda_{j},
\end{gathered}
$$

where $w_{0}=U_{0}$ and $\lambda_{j}$ is for the moment an arbitrary but fixed real number.

Since $f_{j} \in L^{2}(0,1)$, the Lax-Milgram lemma implies, as it is well known, the existence and uniqueness of a solution $w_{j} \in H^{2}(0,1)$ to the elliptic problem (3.5) provided that the previous function $w_{j-1}$ is already known. Thus, starting with $j=1$, this iterative procedure yields the following lemma.

Lemma 3.1. For all $n \geqslant 1$ and for all $\lambda_{j} \in \mathbb{R}$, the auxiliary problems (3.5), $j=$ $1, \ldots, n$, have unique solutions $w_{j} \in H^{2}(0,1)$.

To emphasize the fact that $w_{j}$ depends on $\lambda_{j}$, we will write $w_{j}\left(\cdot, \lambda_{j}\right)$ instead of $w_{j}$. We now introduce, for each $j=1, \ldots, n$, the real function

$$
\Phi_{j}\left(\lambda_{j}\right):=\int_{0}^{1} w_{j}\left(x, \lambda_{j}\right) d x-E_{j} .
$$


We remark that $w_{j}\left(\cdot, \lambda_{j}\right)$ will be a solution to problem (3.2)-(3.4) if and only if $\lambda_{j}$ is a real root of $\Phi_{j}$ so that to establish the existence of a unique solution to (3.2)-(3.4), it is sufficient to show that $\Phi_{j}$ admits exactly one real root. We then express $w_{j}\left(\cdot, \lambda_{j}\right)$ in terms of $\lambda_{j}$. For this, we introduce a new unknown function $v_{j}$ by

$$
w_{j}\left(x, \lambda_{j}\right)=v_{j}(x)+\frac{\lambda_{j}-\alpha_{j}}{2} x^{2}+\alpha_{j} x,
$$

then an easy computation shows that $v_{j}$ thus defined in (3.7) is a solution to the problem

$$
\begin{gathered}
-v_{j}^{\prime \prime}+\frac{1}{h} v_{j}=f_{j}+\frac{1}{h} w_{j-1}+\alpha_{j}\left(\frac{1}{2 h} x^{2}-\frac{1}{h} x-1\right)+\lambda_{j}\left(1-\frac{1}{2 h} x^{2}\right), \quad x \in(0,1), \\
v_{j}^{\prime}(0)=v_{j}^{\prime}(1)=0 .
\end{gathered}
$$

Consequently, $v_{j}$ is the superposition of $\hat{v}_{j}$ and $\tilde{v}_{j}$ which are, respectively, solutions of the following problems

$$
\begin{gathered}
-\hat{v}_{j}^{\prime \prime}+\frac{1}{h} \hat{v}_{j}=f_{j}+\frac{1}{h} w_{j-1}+\alpha_{j}\left(\frac{1}{2 h} x^{2}-\frac{1}{h} x-1\right), \quad x \in(0,1), \\
\hat{v}_{j}^{\prime}(0)=\widehat{v}_{j}^{\prime}(1)=0 \\
-\tilde{v}_{j}^{\prime \prime}+\frac{1}{h} \widetilde{v}_{j}=\lambda_{j}\left(1-\frac{1}{2 h} x^{2}\right), \quad x \in(0,1), \\
\tilde{v}_{j}^{\prime}(0)=\tilde{v}_{j}^{\prime}(1)=0 .
\end{gathered}
$$

Obviously, only $\tilde{v}_{j}$ depends on $\lambda_{j}$. Applying the "variation of parameters method," we easily obtain

$$
\tilde{v}_{j}(x)=\lambda_{j}\left[\frac{\sqrt{h}}{\sinh (1 / \sqrt{h})} \cosh \left(\frac{x}{\sqrt{h}}\right)-\frac{x^{2}}{2}\right], \quad j=1, \ldots, n,
$$

and substituting in (3.7), we get

$$
w_{j}\left(x, \lambda_{j}\right)=\hat{v}_{j}(x)+\alpha_{j} x\left(1-\frac{1}{2} x\right)+\lambda_{j}\left[\frac{\sqrt{h}}{\sinh (1 / \sqrt{h})} \cosh \left(\frac{x}{\sqrt{h}}\right)\right]
$$

so that the function (3.6) can be written in the form

$$
\Phi_{j}\left(\lambda_{j}\right)=h \lambda_{j}+\int_{0}^{1}\left[\hat{v}_{j}(x)+\alpha_{j} x\left(1-\frac{x}{2}\right)\right] d x-E_{j},
$$


906 On the diffusion equation with an integral condition

which proves that $\Phi_{j}$ possesses a unique root $\bar{\lambda}_{j} \in \mathbb{R}$ given by

$$
\bar{\lambda}_{j}=\frac{1}{h}\left\{E_{j}-\int_{0}^{1}\left[\hat{v}_{j}(x)+\alpha_{j} x\left(1-\frac{x}{2}\right)\right] d x\right\} .
$$

Thus, we have just proved the following theorem.

Theorem 3.2. For all $n \geqslant 1$ and for all $j=1, \ldots, n$, problem (3.2)-(3.4) admits a unique solution $u_{j}$ in $H^{2}(0,1)$. Moreover,

$$
u_{j}(x)=w_{j}\left(x, \bar{\lambda}_{j}\right), \quad x \in(0,1)
$$

where $w_{j}\left(\cdot, \bar{\lambda}_{j}\right)$ is the solution of (3.5) with $\bar{\lambda}_{j}$ given by (3.13).

We can now introduce the Rothe function $u^{(n)}: I \rightarrow H^{2}(0,1)$ obtained from the functions $u_{j}$ by piecewise linear interpolation with respect to time

$$
u^{(n)}(t)=u_{j-1}+\delta u_{j}\left(t-t_{j-1}\right), \quad t \in\left[t_{j-1}, t_{j}\right], j=1, \ldots, n,
$$

as well as the step function $\bar{u}^{(n)}: I \rightarrow H^{2}(0,1)$ defined as follows:

$$
\bar{u}^{(n)}(t)= \begin{cases}u_{j}, & \text { if } t \in\left(t_{j-1}, t_{j}\right], j=1, \ldots, n, \\ U_{0}, & \text { if } t=0 .\end{cases}
$$

The functions $u^{(n)}$ and $\bar{u}^{(n)}$ are intended to be approximations of the solution of our problem (2.2)-(2.5) in some suitable function space. To confirm this fact, we derive some a priori estimates for $u_{j}$ and $\delta u_{j}$.

We first work with the following special case.

\section{Case of homogeneous boundary conditions}

Throughout this section, we assume that

$$
\alpha(t)=E(t)=0 \quad \forall t \in I .
$$

Then, for each $j=1, \ldots, n$, problem (3.2)-(3.4) is written as follows:

$$
\begin{gathered}
\delta u_{j}-u_{j}^{\prime \prime}=f_{j}, \quad x \in(0,1), \\
u_{j}^{\prime}(0)=0, \\
\int_{0}^{1} u_{j}(x) d x=0,
\end{gathered}
$$


and assumption $\left(\mathrm{A}_{4}\right)$ becomes

$$
U_{0}^{\prime}(0)=0, \quad \int_{0}^{1} U_{0}(x) d x=0
$$

\subsection{A priori estimates for the approximations}

Lemma 4.1. There exists $C>0$ such that, for all $n \geqslant 1$, the solutions $u_{j}$ of the discretized problems (4.2)-(4.4), $j=1, \ldots, n$, satisfy the estimates

$$
\begin{gathered}
\left\|u_{j}\right\| \leqslant C, \\
\left\|\delta u_{j}\right\|_{B_{2}^{1}(0,1)} \leqslant C .
\end{gathered}
$$

Proof. As it will be seen later, the first estimate follows from the second one, hence we begin by this latter.

Taking, for all $j=1, \ldots, n$, the inner product in $B_{2}^{1}(0,1)$ of $(4.2)$ with any $\phi \in$ $V$, we get

$$
\left(\delta u_{j}, \phi\right)_{B_{2}^{1}(0,1)}-\left(u_{j}^{\prime \prime}, \phi\right)_{B_{2}^{1}(0,1)}=\left(f_{j}, \phi\right)_{B_{2}^{1}(0,1)} .
$$

It follows from (4.3) that

$$
\begin{aligned}
\left(u_{j}^{\prime \prime}, \phi\right)_{B_{2}^{1}(0,1)} & =\int_{0}^{1} \mathfrak{J}_{x}\left(u_{j}^{\prime \prime}\right) \mathfrak{I}_{x} \phi d x \\
& =\int_{0}^{1}\left[u_{j}^{\prime}(x)-u_{j}^{\prime}(0)\right] \mathfrak{J}_{x} \phi d x \\
& =\int_{0}^{1} u_{j}^{\prime}(x) \mathfrak{I}_{x} \phi d x
\end{aligned}
$$

so that the standard integration by parts leads to

$$
\left(u_{j}^{\prime \prime}, \phi\right)_{B_{2}^{1}(0,1)}=\left.u_{j}(x) \mathfrak{I}_{x} \phi\right|_{x=0} ^{x=1}-\int_{0}^{1} u_{j} \phi d x=-\left(u_{j}, \phi\right)
$$

since $\int_{0}^{1} \phi(x) d x=0$. Substituting into (4.8), we finally obtain

$$
\left(\delta u_{j}, \phi\right)_{B_{2}^{1}(0,1)}+\left(u_{j}, \phi\right)=\left(f_{j}, \phi\right)_{B_{2}^{1}(0,1)}
$$

for all $\phi \in V$.

Consider the identity

$$
\left(\delta u_{1}, \phi\right)_{B_{2}^{1}(0,1)}+h\left(\delta u_{1}, \phi\right)=\left(f_{1}, \phi\right)_{B_{2}^{1}(0,1)}-\left(U_{0}, \phi\right)
$$


908 On the diffusion equation with an integral condition

which results from (4.11) with $j=1$. Performing an integration by parts, we get

$$
\begin{aligned}
\left(U_{0}, \phi\right) & =\int_{0}^{1} U_{0}(x)\left(\mathfrak{I}_{x} \phi\right)^{\prime} d x \\
& =\left.U_{0}(x) \mathfrak{I}_{x} \phi\right|_{x=0} ^{x=1}-\int_{0}^{1} U_{0}^{\prime}(x) \mathfrak{J}_{x} \phi d x \\
& =-\int_{0}^{1} U_{0}^{\prime}(x) \mathfrak{J}_{x} \phi d x,
\end{aligned}
$$

but assumption $\left(A_{3}\right)$ and the first condition in (4.5) yield

$$
\mathfrak{I}_{x}\left(U_{0}^{\prime \prime}\right)=U_{0}^{\prime}(x) \quad \forall x \in(0,1)
$$

from which it follows that

$$
\left(U_{0}, \phi\right)=-\int_{0}^{1} \mathfrak{J}_{x}\left(U_{0}^{\prime \prime}\right) \mathfrak{I}_{x} \phi d x=-\left(U_{0}^{\prime \prime}, \phi\right)_{B_{2}^{1}(0,1)} .
$$

Substituting in the right-hand side of (4.12), (4.15) becomes

$$
\left(\delta u_{1}, \phi\right)_{B_{2}^{1}(0,1)}+h\left(\delta u_{1}, \phi\right)=\left(f_{1}+U_{0}^{\prime \prime}, \phi\right)_{B_{2}^{1}(0,1)} .
$$

Since $\delta u_{1}$ is an element of $V$ in view of (4.4) with $j=1$, the second condition in (4.5), and assumption $\left(A_{3}\right)$, it may be employed as a test function in (4.16) to get with the aid of Cauchy-Schwarz inequality

$$
\left\|\delta u_{1}\right\|_{B_{2}^{1}(0,1)}^{2}+h\left\|\delta u_{1}\right\|^{2} \leqslant\left(\left\|f_{1}\right\|_{B_{2}^{1}(0,1)}+\left\|U_{0}^{\prime \prime}\right\|_{B_{2}^{1}(0,1)}\right)\left\|\delta u_{1}\right\|_{B_{2}^{1}(0,1)}
$$

hence

$$
\left\|\delta u_{1}\right\|_{B_{2}^{1}(0,1)} \leqslant\|f\|_{C\left(I, B_{2}^{1}(0,1)\right)}+\left\|U_{0}^{\prime \prime}\right\|_{B_{2}^{1}(0,1)} .
$$

Now we take the difference of the relations (4.11) and (4.11) with $j$ replaced by $j-1, j=2, \ldots, n$, applied to the test function $\phi=\delta u_{j}$ which is in $V$ by virtue of (4.4) and (4.4) with $j$ replaced by $j-1$; we have

$$
\left\|\delta u_{j}\right\|_{B_{2}^{1}(0,1)}^{2}+\frac{1}{h}\left\|u_{j}-u_{j-1}\right\|^{2}=\left(f_{j}-f_{j-1}, \delta u_{j}\right)_{B_{2}^{1}(0,1)}+\left(\delta u_{j-1}, \delta u_{j}\right)_{B_{2}^{1}(0,1)} .
$$

Applying Cauchy-Schwarz inequality and omitting the second term in the lefthand side, we obtain

$$
\left\|\delta u_{j}\right\|_{B_{2}^{1}(0,1)}^{2} \leqslant\left\|f_{j}-f_{j-1}\right\|_{B_{2}^{1}(0,1)}\left\|\delta u_{j}\right\|_{B_{2}^{1}(0,1)}+\left\|\delta u_{j-1}\right\|_{B_{2}^{1}(0,1)}\left\|\delta u_{j}\right\|_{B_{2}^{1}(0,1)} .
$$


Hence, invoking assumption $\left(A_{1}\right)$, we have

$$
\left\|\delta u_{j}\right\|_{B_{2}^{1}(0,1)} \leqslant l h+\left\|\delta u_{j-1}\right\|_{B_{2}^{1}(0,1)}
$$

so that, by an iterative procedure, we may arrive at

$$
\left\|\delta u_{j}\right\|_{B_{2}^{1}(0,1)} \leqslant l(j-1) h+\left\|\delta u_{1}\right\|_{B_{2}^{1}(0,1)} .
$$

Finally, in light of (4.18), we obtain

$$
\left\|\delta u_{j}\right\|_{B_{2}^{1}(0,1)} \leqslant l T+\|f\|_{C\left(I, B_{2}^{1}(0,1)\right)}+\left\|U_{0}^{\prime \prime}\right\|_{B_{2}^{1}(0,1)}
$$

for every $j=1, \ldots, n$. This proves (4.7) with $C=l T+\|f\|_{C\left(I, B_{2}^{1}(0,1)\right)}+\left\|U_{0}^{\prime \prime}\right\|_{B_{2}^{1}(0,1)}$. Next, we majorize $\left\|u_{j}\right\|$. The application of the formula

$$
\left(u_{j}, u_{j}-u_{j-1}\right)=\frac{1}{2}\left(\left\|u_{j}\right\|^{2}-\left\|u_{j-1}\right\|^{2}+\left\|u_{j}-u_{j-1}\right\|^{2}\right)
$$

to (4.11) with $\phi=u_{j}-u_{j-1}$ as test functions, $j=1, \ldots, n$, yields

$$
h\left\|\delta u_{j}\right\|_{B_{2}^{1}(0,1)}^{2}+\frac{1}{2}\left\|u_{j}-u_{j-1}\right\|^{2}+\frac{1}{2}\left\|u_{j}\right\|^{2}=\left(f_{j}, u_{j}-u_{j-1}\right)_{B_{2}^{1}(0,1)}+\frac{1}{2}\left\|u_{j-1}\right\|^{2} .
$$

Omitting the first two terms in the left-hand side and using Cauchy-Schwarz inequality, we obtain

$$
\left\|u_{j}\right\|^{2} \leqslant 2\left\|f_{j}\right\|_{B_{2}^{1}(0,1)} \mid\left\|u_{j}-u_{j-1}\right\|_{B_{2}^{1}(0,1)}+\left\|u_{j-1}\right\|^{2} .
$$

So, in consideration of (4.23), we have

$$
\left\|u_{j}\right\|^{2} \leqslant 2 h\|f\|_{C\left(I, B_{2}^{1}(0,1)\right)}\left(l T+\|f\|_{C\left(I, B_{2}^{1}(0,1)\right)}+\left\|U_{0}^{\prime \prime}\right\|_{B_{2}^{1}(0,1)}\right)+\left\|u_{j-1}\right\|^{2} .
$$

Iterating this inequality, we may obtain

$$
\left\|u_{j}\right\|^{2} \leqslant 2 j h\|f\|_{C\left(I, B_{2}^{1}(0,1)\right)}\left(l T+\|f\|_{C\left(I, B_{2}^{1}(0,1)\right)}+\left\|U_{0}^{\prime \prime}\right\|_{B_{2}^{1}(0,1)}\right)+\left\|U_{0}\right\|^{2} .
$$

Hence, the estimate (4.6) follows with

$$
C=\sqrt{2 T\|f\|_{C\left(I, B_{2}^{1}(0,1)\right)}\left(l T+\|f\|_{C\left(I, B_{2}^{1}(0,1)\right)}+\left\|U_{0}^{\prime \prime}\right\|_{B_{2}^{1}(0,1)}\right)+\left\|U_{0}\right\|^{2}}
$$

and so our proof is complete. 
910 On the diffusion equation with an integral condition

As a consequence of Lemma 4.1 and the definition of $u^{(n)}$ and $\bar{u}^{(n)}$, we obtain the following corollary.

Corollary 4.2. For all $n \geqslant 1$, the functions $u^{(n)}$ and $\bar{u}^{(n)}$ satisfy the estimates

$$
\begin{gathered}
\left\|u^{(n)}(t)\right\| \leqslant C, \quad\left\|\bar{u}^{(n)}(t)\right\| \leqslant C, \quad \forall t \in I, \\
\left\|\frac{d u^{(n)}}{d t}(t)\right\|_{B_{2}^{1}(0,1)} \leqslant C \quad \text { for a.e. } t \in I, \\
\left\|\bar{u}^{(n)}(t)-u^{(n)}(t)\right\|_{B_{2}^{1}(0,1)} \leqslant \frac{C}{n} \quad \forall t \in I, \\
\left\|u^{(n)}(t)-u^{(n)}\left(t^{\prime}\right)\right\|_{B_{2}^{1}(0,1)} \leqslant C\left|t-t^{\prime}\right| \quad \forall t, t^{\prime} \in I .
\end{gathered}
$$

Proof. Estimates (4.30) follow immediately from estimate (4.6) with the same constant, whereas estimate (4.31) is an easy consequence of estimate (4.7), also with the same constant, noting that we have

$$
\frac{d u^{(n)}}{d t}(t)=\delta u_{j}, \quad t \in\left(t_{j-1}, t_{j}\right], 1 \leqslant j \leqslant n .
$$

For estimate (4.32), it suffices to see that we have

$$
\bar{u}^{(n)}(t)-u^{(n)}(t)=\left(t_{j}-t\right) \delta u_{j}, \quad t \in\left(t_{j-1}, t_{j}\right], 1 \leqslant j \leqslant n,
$$

and consequently,

$$
\left\|\bar{u}^{(n)}(t)-u^{(n)}(t)\right\|_{B_{2}^{1}(0,1)} \leqslant h \max _{1 \leqslant j \leqslant n}\left\|\delta u_{j}\right\|_{B_{2}^{1}(0,1)} \quad \forall t \in I .
$$

Hence, applying estimate (4.7), we get (4.32) with

$$
C=T\left(l T+\|f\|_{C\left(I, B_{2}^{1}(0,1)\right)}+\left\|U_{0}^{\prime \prime}\right\|_{B_{2}^{1}(0,1)}\right) .
$$

Finally, using the inequality

$$
\left\|u^{(n)}(t)-u^{(n)}\left(t^{\prime}\right)\right\|_{B_{2}^{1}(0,1)} \leqslant\left|\int_{t^{\prime}}^{t}\left\|\frac{d u^{(n)}}{d s}(s)\right\|_{B_{2}^{1}(0,1)} d s\right|,
$$

which holds for all $t, t^{\prime} \in I$, we obtain (4.33) in view of estimate (4.31). 
4.2. Convergence and existence result. Define the step function $\bar{f}^{(n)}: I \rightarrow$ $L^{2}(0,1)$ by setting

$$
\bar{f}^{(n)}(t)= \begin{cases}f_{j}, & \text { if } t \in\left(t_{j-1}, t_{j}\right], j=1, \ldots, n, \\ f_{0}, & \text { if } t=0\end{cases}
$$

Then, for all $v \in L^{2}(I, V)$, the variational equation (4.11) may be written in terms of $u^{(n)}, \bar{u}^{(n)}$, and $\bar{f}^{(n)}$ as follows:

$$
\left(\frac{d u^{(n)}}{d t}(t), v(t)\right)_{B_{2}^{1}(0,1)}+\left(\bar{u}^{(n)}(t), v(t)\right)=\left(\bar{f}^{(n)}(t), v(t)\right)_{B_{2}^{1}(0,1)} \quad \text { for a.e. } t \in I
$$

Integrating this formula over $I$, we obtain the following approximation: scheme

$$
\begin{array}{r}
\int_{I}\left(\frac{d u^{(n)}}{d t}(t), v(t)\right)_{B_{2}^{1}(0,1)} d t+\int_{I}\left(\bar{u}^{(n)}(t), v(t)\right) d t \\
=\int_{I}\left(\bar{f}^{(n)}(t), v(t)\right)_{B_{2}^{1}(0,1)} d t \quad \forall v \in L^{2}(I, V),
\end{array}
$$

and we propose to establish the convergence of it to the weak formulation of problem (2.2)-(2.5), given by (2.15). The results of Corollary 4.2 are the basis for the following convergence assertions for the Rothe approximations.

Theorem 4.3. There exists a function $u \in L^{2}(I, V) \cap C\left(I, B_{2}^{1}(0,1)\right)$ with $d u / d t \in$ $L^{2}\left(I, B_{2}^{1}(0,1)\right)$ and subsequences $\left\{u^{\left(n_{k}\right)}\right\}_{k} \subseteq\left\{u^{(n)}\right\}_{n},\left\{\bar{u}^{\left(n_{k}\right)}\right\}_{k} \subseteq\left\{\bar{u}^{(n)}\right\}_{n}$ such that

$$
\begin{gathered}
u^{\left(n_{k}\right)} \rightarrow u \quad \text { in } L^{2}(I, V), \\
\bar{u}^{\left(n_{k}\right)} \rightarrow u \quad \text { in } L^{2}(I, V), \\
\frac{d u^{\left(n_{k}\right)}}{d t} \rightarrow \frac{d u}{d t} \quad \text { in } L^{2}\left(I, B_{2}^{1}(0,1)\right) .
\end{gathered}
$$

Proof. Estimates (4.30) imply the uniform boundedness of $\left\{u^{(n)}\right\}_{n}$ and $\left\{\bar{u}^{(n)}\right\}_{n}$ in $L^{2}(I, V)$ with respect to $n$. Therefore, by extracting some subsequences $\left\{u^{\left(n_{k}\right)}\right\}_{k}$ and $\left\{\bar{u}^{\left(n_{k}\right)}\right\}_{k}$, they converge in the weak topology to some functions $u$ and $\bar{u}$ in $L^{2}(I, V)$, as $k \rightarrow \infty$, respectively. We show that $\bar{u}$ coincides with $u$. Since $V \hookrightarrow$ $B_{2}^{1}(0,1)$, we have also

$$
\begin{array}{ll}
u^{\left(n_{k}\right)}-u & \text { in } L^{2}\left(I, B_{2}^{1}(0,1)\right), \\
\bar{u}^{\left(n_{k}\right)}-\bar{u} & \text { in } L^{2}\left(I, B_{2}^{1}(0,1)\right)
\end{array}
$$


912 On the diffusion equation with an integral condition

From the equality $\bar{u}^{\left(n_{k}\right)}-u=\left(\bar{u}^{\left(n_{k}\right)}-u^{\left(n_{k}\right)}\right)+\left(u^{\left(n_{k}\right)}-u\right)$, it follows by means of (4.32) that

$$
\begin{aligned}
\left|\left(\bar{u}^{\left(n_{k}\right)}-u, v\right)_{L^{2}\left(I, B_{2}^{1}(0,1)\right)}\right| \leqslant & \left\|\bar{u}^{\left(n_{k}\right)}-\left.u^{\left(n_{k}\right)}\right|_{L^{2}\left(I, B_{2}^{1}(0,1)\right)}\right\| v \|_{L^{2}\left(I, B_{2}^{1}(0,1)\right)} \\
& +\left|\left(u^{\left(n_{k}\right)}-u, v\right)_{L^{2}\left(I, B_{2}^{1}(0,1)\right)}\right| \\
\leqslant & \frac{C}{n_{k}}\|v\|_{L^{2}\left(I, B_{2}^{1}(0,1)\right)}+\left|\left(u^{\left(n_{k}\right)}-u, v\right)_{L^{2}\left(I, B_{2}^{1}(0,1)\right)}\right|
\end{aligned}
$$

for all $v \in L^{2}\left(I, B_{2}^{1}(0,1)\right)$, so that by passing to the limit as $k \rightarrow \infty$, we get, in view of (4.45), $\left|\left(\bar{u}^{\left(n_{k}\right)}-u, v\right)_{L^{2}\left(I, B_{2}^{1}(0,1)\right)}\right| \rightarrow 0$, that is, $\bar{u}^{\left(n_{k}\right)} \rightarrow u$ in $L^{2}\left(I, B_{2}^{1}(0,1)\right)$; consequently $u=\bar{u}$ holds. Hence, we obtain (4.43). On the other hand, according to $(4.31),\left\{d u^{(n)} / d t\right\}_{n}$ is bounded in $L^{2}\left(I, B_{2}^{1}(0,1)\right)$. Thus, there is a subsequence $\left\{d u^{\left(n_{k}\right)} / d t\right\}_{k}$ and some $w \in L^{2}\left(I, B_{2}^{1}(0,1)\right)$ such that

$$
\frac{d u^{\left(n_{k}\right)}}{d t} \rightarrow w \quad \text { in } L^{2}\left(I, B_{2}^{1}(0,1)\right)
$$

It remains to show that $w$ equals $d u / d t$ in $L^{2}\left(I, B_{2}^{1}(0,1)\right)$. For this, we consider the equality

$$
u^{\left(n_{k}\right)}(t)-U_{0}=\int_{0}^{t} \frac{d u^{\left(n_{k}\right)}(s)}{d s} d s \quad \forall t \in I
$$

which ensues from the construction of $u^{(n)}$ and (3.1). It follows due to (4.45) and (4.48) that [23, page 207]

$$
u(t)=U_{0}+\int_{0}^{t} w(s) d s \quad \forall t \in I
$$

(Bochner integral in $\left.B_{2}^{1}(0,1)\right)$ which implies [16, Lemmas 1.3.2(iii) and 1.3.6(i)] that $u$ is in $C\left(I, B_{2}^{1}(0,1)\right)$ and even (strongly) differentiable a.e. in $I$ with $d u / d t=$ $w$ in $L^{2}\left(I, B_{2}^{1}(0,1)\right)$, which was to be shown.

Now we are prepared to state an existence theorem.

Theorem 4.4. The limit function $u$ is the unique weak solution to problem (2.2)(2.5) in the case of (4.1) in the sense of Definition 2.1.

Proof. Note that in light of what precedes, we have $u \in L^{2}(I, V) \cap C\left(I, B_{2}^{1}(0,1)\right)$, and consequently $u$ fulfils the integral condition (2.5) with $E(t) \equiv 0$ since $u(t)$ belongs to $V$ for a.e. $t \in I$. Moreover, according to $(4.50), u(0)=U_{0}$ holds, so the initial condition (2.3) is also fulfilled. To see that $u$ obeys the weak formulation of problem (2.2)-(2.5), we will show that approximation scheme (4.41) approaches, for the subsequence $\left\{n_{k}\right\}_{k} \subseteq\{n\}_{n}$, the integral relation (2.15) with 
$\gamma(t) \equiv 0$. We note that assumption $\left(\mathrm{A}_{1}\right)$ implies that

$$
\left\|\bar{f}^{(n)}(t)-f(t)\right\|_{B_{2}^{1}(0,1)} \leqslant \frac{C}{n} \quad \text { a.e. in } I,
$$

from which we deduce easily that

$$
\left\|\bar{f}^{(n)}-f\right\|_{L^{2}\left(I, B_{2}^{1}(0,1)\right)} \leqslant \frac{C}{n} \longrightarrow 0 \quad \text { as } n \longrightarrow \infty,
$$

that is,

$$
\bar{f}^{(n)} \longrightarrow f \quad \text { in } L^{2}\left(I, B_{2}^{1}(0,1)\right) .
$$

Finally, a limiting process $n=n_{k} \rightarrow \infty$ in approximation scheme (4.41) by means of the convergence properties (4.43), (4.44), and (4.53) immediately yields

$$
\begin{aligned}
\int_{I}\left(\frac{d u}{d t}(t),\right. & v(t))_{B_{2}^{1}(0,1)} d t+\int_{I}(u(t), v(t)) d t \\
& =\int_{I}(f(t), v(t))_{B_{2}^{1}(0,1)} d t \quad \forall v \in L^{2}(I, V) .
\end{aligned}
$$

Thus, $u$ weakly solves problem (2.2)-(2.5). The uniqueness can be shown in a standard way. Indeed, if $u^{*}$ and $u^{* *}$ are two weak solutions of (2.2)-(2.5), then the difference $u:=u^{*}-u^{* *}$ satisfies

$$
\int_{I}\left(\frac{d u}{d t}(t), v(t)\right)_{B_{2}^{1}(0,1)} d t+\int_{I}(u(t), v(t)) d t=0 \quad \forall v \in L^{2}(I, V)
$$

besides, $u(0)=0$ holds.

For every fixed $t^{0} \in I$, we define

$$
v(t)= \begin{cases}u(t), & 0 \leqslant t \leqslant t^{0} \\ 0, & t^{0}<t \leqslant T\end{cases}
$$

which obviously belongs to $L^{2}(I, V)$. Using (4.56) as a test function in the last integral relation, we obtain

$$
\int_{0}^{t^{0}}\left(\frac{d u}{d t}(t), u(t)\right)_{B_{2}^{1}(0,1)} d t+\int_{0}^{t^{0}}\|u(t)\|^{2} d t=0
$$


914 On the diffusion equation with an integral condition

or

$$
\int_{0}^{t^{0}}\left(\frac{d u}{d t}(t), u(t)\right)_{B_{2}^{1}(0,1)} d t \leqslant 0
$$

so that, in view of the formula $(d / d t)\|u(t)\|_{B_{2}^{1}(0,1)}^{2}=2((d u / d t)(t), u(t))_{B_{2}^{1}(0,1)}$ and due to $u(0)=0$, we get $\left\|u\left(t^{0}\right)\right\|_{B_{2}^{1}(0,1)}^{2} \leqslant 0$. Consequently, $u(t)=0$ for all $t \in I$, meaning that $u^{*}=u^{* *}$, that is, uniqueness, which achieves the proof.

Remark 4.5. As a consequence of the uniqueness of the weak solution of (2.2)(2.5), we conclude, by the usual argument, that the convergence assertions for the Rothe approximations in Theorem 4.3 hold for the whole sequence $\left\{u^{(n)}\right\}_{n}$.

Actually, we may state the following uniform convergence assertion.

THEOREM 4.6. The sequence $\left\{u^{(n)}\right\}_{n}$ converges in the norm of the space $C\left(I, B_{2}^{1}(0\right.$, 1)) to the solution $u$ of (2.2)-(2.5), and the error estimate

$$
\left\|u^{(n)}-u\right\|_{C\left(I, B_{2}^{1}(0,1)\right)} \leqslant C h^{1 / 2}
$$

takes place with some positive constant.

Proof. We prove that $\left\{u^{(n)}\right\}_{n}$ is a Cauchy sequence in $C\left(I, B_{2}^{1}(0,1)\right)$. For this purpose, we estimate the difference $u^{(n)}-u^{(m)}$, where $u^{(n)}$ and $u^{(m)}$ are Rothe approximations corresponding to the step lengths $h_{n}=T / n$ and $h_{m}=T / m$, respectively. Estimating the identity

$$
\left(\bar{u}^{(n)}(t), v(t)\right)=\left(\bar{f}^{(n)}(t)-\frac{d u^{(n)}}{d t}(t), v(t)\right)_{B_{2}^{1}(0,1)} \quad \text { a.e. in } I, \forall v \in L^{2}(I, V),
$$

which results from (4.40), we obtain, owing to (4.31),

$$
\begin{aligned}
\left|\left(\bar{u}^{(n)}(t), v(t)\right)\right| & \leqslant\left(\left\|\bar{f}^{(n)}(t)\right\|_{B_{2}^{1}(0,1)}+\left\|\frac{d u^{(n)}}{d t}(t)\right\|_{B_{2}^{1}(0,1)}\right)\|v(t)\|_{B_{2}^{1}(0,1)} \\
& \leqslant C_{1}\|v(t)\|_{B_{2}^{1}(0,1)} \quad \text { a.e. in } I, \forall v \in L^{2}(I, V),
\end{aligned}
$$

with $C_{1}=l T+2\|f\|_{C\left(I, B_{2}^{1}(0,1)\right)}+\left\|U_{0}^{\prime \prime}\right\|_{B_{2}^{1}(0,1)}$. Now we take the difference of the relations (4.40) and (4.40) written for $m$ with $m \neq n$ and test it with $v=u^{(n)}$ $u^{(m)}\left(\in L^{2}(I, V)\right)$. This yields

$$
\begin{aligned}
\left(\frac{d}{d t}\left(u^{(n)}(t)-u^{(m)}(t)\right), u^{(n)}(t)-u^{(m)}(t)\right)_{B_{2}^{1}(0,1)} & \\
& +\left(\bar{u}^{(n)}(t)-\bar{u}^{(m)}(t), u^{(n)}(t)-u^{(m)}(t)\right) \\
= & \left(\bar{f}^{(n)}(t)-\bar{f}^{(m)}(t), u^{(n)}(t)-u^{(m)}(t)\right)_{B_{2}^{1}(0,1)} \quad \text { a.e. in } I
\end{aligned}
$$


or after some rearrangement,

$$
\begin{aligned}
\frac{1}{2} \frac{d}{d t} \| & u^{(n)}(t)-u^{(m)}(t)\left\|_{B_{2}^{1}(0,1)}^{2}+\right\| \bar{u}^{(n)}(t)-\bar{u}^{(m)}(t) \|^{2} \\
= & \left(\bar{u}^{(n)}(t)-\bar{u}^{(m)}(t), \bar{u}^{(n)}(t)-\bar{u}^{(m)}(t)-u^{(n)}(t)+u^{(m)}(t)\right) \\
& +\left(\bar{f}^{(n)}(t)-\bar{f}^{(m)}(t), u^{(n)}(t)-u^{(m)}(t)\right)_{B_{2}^{1}(0,1)} \quad \text { a.e. in } I .
\end{aligned}
$$

Ignoring the second term in the left-hand side which is clearly positive, we estimate, with the aid of (4.61), (4.30), and (4.32),

$$
\begin{aligned}
\frac{1}{2} \frac{d}{d t}\left\|u^{(n)}(t)-u^{(m)}(t)\right\|_{B_{2}^{1}(0,1)}^{2} & \\
\leqslant & 2 C_{1}\left(\left\|\bar{u}^{(n)}(t)-u^{(n)}(t)\right\|_{B_{2}^{1}(0,1)}+\left\|\bar{u}^{(m)}(t)-u^{(m)}(t)\right\|_{B_{2}^{1}(0,1)}\right) \\
& +\left\|\bar{f}^{(n)}(t)-\bar{f}^{(m)}(t)\right\|_{B_{2}^{1}(0,1)}\left\|u^{(n)}(t)-u^{(m)}(t)\right\|_{B_{2}^{1}(0,1)} \\
\leqslant & 2 C_{1}^{2}\left(h_{n}+h_{m}\right)+2 C_{2}\left\|\bar{f}^{(n)}(t)-\bar{f}^{(m)}(t)\right\|_{B_{2}^{1}(0,1)}
\end{aligned}
$$

with

$$
C_{2}=\sqrt{2 T\|f\|_{C\left(I, B_{2}^{1}(0,1)\right)}\left(l T+\|f\|_{C\left(I, B_{2}^{1}(0,1)\right)}+\left\|U_{0}^{\prime \prime}\right\|_{B_{2}^{1}(0,1)}\right)+\left\|U_{0}\right\|^{2}} .
$$

Assume now that $n<m$, then $h_{m}<h_{n}$. For all $t \in(0, T]$, there exist two integers $k=k(n)$ and $i=i(m)$ such that $t \in\left(t_{k-1}, t_{k}\right] \cap\left(t_{i-1}, t_{i}\right]$. Hence, from assumption $\left(\mathrm{A}_{1}\right)$, it follows that

$$
\left\|\bar{f}^{(n)}(t)-\bar{f}^{(m)}(t)\right\|_{B_{2}^{1}(0,1)}=\left\|f\left(t_{k}\right)-f\left(t_{i}\right)\right\|_{B_{2}^{1}(0,1)} \leqslant l\left|t_{k}-t_{i}\right| .
$$

Consequently,

$$
\left\|\bar{f}^{(n)}(t)-\bar{f}^{(m)}(t)\right\|_{B_{2}^{1}(0,1)} \leqslant l h_{n} \quad \forall t \in I .
$$

Substituting into (4.64) and integrating the resulting inequality over $(0, t) \subset I$, invoking the fact that $u^{(n)}(0)=u^{(m)}(0)=U_{0}$, we get

$$
\begin{aligned}
\left\|u^{(n)}(t)-u^{(m)}(t)\right\|_{B_{2}^{1}(0,1)}^{2} & \leqslant 4\left(C_{1}^{2}\left(h_{n}+h_{m}\right)+C_{2} l h_{n}\right) t \\
& \leqslant 4\left(C_{1}^{2}\left(h_{n}+h_{m}\right)+C_{2} l h_{n}\right) T
\end{aligned}
$$

so that

$$
\left\|u^{(n)}-u^{(m)}\right\|_{C\left(I, B_{2}^{1}(0,1)\right)} \leqslant 2 \sqrt{T\left(C_{1}^{2}\left(h_{n}+h_{m}\right)+C_{2} l h_{n}\right)} .
$$


916 On the diffusion equation with an integral condition

This implies that $\left\{u^{(n)}\right\}_{n}$ is a Cauchy sequence in the Banach space $C\left(I, B_{2}^{1}(0,1)\right)$, and hence it converges in the norm of this latter to some function which is not other than $u$. Besides, passing to the limit $m \rightarrow \infty$ in (4.69), we obtain the desired error estimate with $C=2 \sqrt{T\left(C_{1}^{2}+C_{2} l\right)}$ and $h=h_{n}$, which finishes the proof.

Now, we present some additional properties of the obtained solution.

TheOrem 4.7. For the solution $u$ of (2.2)-(2.5), the following assertions hold:

(i) $u \in C^{0,1}\left(I, B_{2}^{1}(0,1)\right) \cap L^{\infty}(I, V)$ and $d u / d t \in L^{\infty}\left(I, B_{2}^{1}(0,1)\right)$;

(ii) $u$ depends continuously upon data $f$ and $U_{0}$; namely,

$$
\|u\|_{C\left(I, B_{2}^{1}(0,1)\right)} \leqslant C\left(\|f\|_{C\left(I, B_{2}^{1}(0,1)\right)}+\left\|U_{0}\right\|_{H^{2}(0,1)}\right)
$$

where $C>0$ depends neither on $f$ nor on $U_{0}$.

Proof. The Lipschitz continuity of $u$ follows immediately from (4.33) and convergence result from Theorem 4.6. From this fact, we have $\|(d u / d t)(t)\|_{B_{2}^{1}(0,1)} \leqslant$ $C$ for a.a. $t \in I$ since $u$ is strongly differentiable, that is, $d u / d t \in L^{\infty}\left(I, B_{2}^{1}(0,1)\right)$. Since the space $L^{\infty}(I, V)$ is the dual space to the separable Banach space $L^{1}(I, V)$, every bounded set in $L^{\infty}(I, V)$ is compact in the weak* topology. From the first estimate in (4.30), we infer that

$$
\max _{t \in I}\left\|u^{(n)}(t)\right\| \leqslant C, \quad \text { uniformly on } n \text {. }
$$

Consequently, there exist $\omega \in L^{\infty}(I, V)$ and a subsequence $\left\{u^{\left(n_{k}\right)}\right\}_{k} \subseteq\left\{u^{(n)}\right\}_{n}$ such that $u^{\left(n_{k}\right)} \stackrel{*}{\rightarrow} \omega$ in $L^{\infty}(I, V)$, from which it follows also that $u^{\left(n_{k}\right)} \rightarrow \omega$ in $L^{2}(I, V) \supset L^{\infty}(I, V)$, and hence due to (4.42), $u=\omega$ is true. Thus $u \in L^{\infty}(I, V)$.

With regard to Theorem 4.7(ii), the first estimate in (4.30) and convergence result from Theorem 4.6 yield

$$
\|u\|_{C\left(I, B_{2}^{1}(0,1)\right)} \leqslant \sqrt{T\|f\|_{C\left(I, B_{2}^{1}(0,1)\right)}\left(l T+\|f\|_{C\left(I, B_{2}^{1}(0,1)\right)}+\left\|U_{0}^{\prime \prime}\right\|_{B_{2}^{1}(0,1)}\right)+\frac{1}{2}\left\|U_{0}\right\|^{2}}
$$

where (2.12) has been used. This shows that the mapping $\left(f, U_{0}\right) \mapsto u$ from $C\left(I, B_{2}^{1}(0,1)\right) \times H^{2}(0,1)$ into $C\left(I, B_{2}^{1}(0,1)\right)$ is bounded on the unit ball $\left\|\left(f, U_{0}\right)\right\|$ $:=\|f\|_{C\left(I, B_{2}^{1}(0,1)\right)}+\left\|U_{0}\right\|_{H^{2}(0,1)} \leqslant 1$ and hence continuous since it is linear. Accordingly, the inequality (4.70) follows for $C=\sqrt{T(l T+1)+1 / 2}$, which expresses the continuous dependence of $u$ on data, so the proof is complete.

At the end of this subsection, we summarize all the obtained results into the following theorem. 
Theorem 4.8. Let assumptions $\left(A_{1}\right),\left(A_{3}\right)$, and $\left(A_{4}\right)$ be fulfilled. Then, problem (2.2)-(2.5) with homogeneous boundary conditions has a unique weak solution $u$ in the sense of Definition 2.1 with additional regularity $u \in C^{0,1}\left(I, B_{2}^{1}(0,1)\right) \cap$ $L^{\infty}(I, V)$ and $d u / d t \in L^{\infty}\left(I, B_{2}^{1}(0,1)\right)$.

The sequence of Rothe functions converges to the solution $u$ in the following sense:

$$
\begin{gathered}
u^{(n)} \rightarrow u \quad \text { in } L^{2}(I, V), \\
u^{(n)} \rightarrow u \quad \text { in } C\left(I, B_{2}^{1}(0,1)\right), \\
\frac{d u^{(n)}}{d t} \rightarrow \frac{d u}{d t} \quad \text { in } L^{2}\left(I, B_{2}^{1}(0,1)\right),
\end{gathered}
$$

as $n \rightarrow \infty$.

Moreover, $u$ depends continuously on the right-hand side of (2.2) and on the initial function.

\section{Case of nonhomogeneous boundary conditions}

We now return to the general case without assuming (4.1). Then, instead of (4.11), we have

$$
\left(\delta u_{j}, \phi\right)_{B_{2}^{1}(0,1)}+\left(u_{j}, \phi\right)=\left(f_{j}, \phi\right)_{B_{2}^{1}(0,1)}-\alpha_{j} \int_{0}^{1} \mathfrak{I}_{x} \phi d x \quad \forall \phi \in V,
$$

but since, here, $u_{j}$ and $\delta u_{j}$ are not in $V$, they are not admissible test functions for the aforesaid identity, which hinders obtaining an analog of the crucial a priori estimate (4.7) and on the basis of which convergence and existence results are established in the previous case. However, in order to exploit the results of the preceding section, we introduce the transform

$$
u_{j}=\tilde{u}_{j}+\gamma_{j}, \quad j=0, \ldots, n,
$$

where

$$
\gamma_{j}(x)=\alpha_{j}\left(x-\frac{1}{2}\right)+E_{j} \quad \forall x \in(0,1), j=0, \ldots, n
$$

Then, noticing that $\gamma_{j}^{\prime}(0)=\alpha_{j}$ and $\int_{0}^{1} \gamma_{j}(x) d x=\left[\alpha_{j}\left(x^{2} / 2-x / 2\right)+E_{j} x\right]_{x=0}^{x=1}=E_{j}$, problems (3.1)-(3.4) are reduced as follows:

$$
\begin{gathered}
\delta \tilde{u}_{j}-\tilde{u}_{j}^{\prime \prime}=f_{j}-\delta \gamma_{j}, \quad j=1, \ldots, n, \\
\tilde{u}_{j}^{\prime}(0)=0, \quad \int_{0}^{1} \tilde{u}_{j}(x) d x=0, \quad \tilde{u}_{0}=U_{0}-\gamma_{0},
\end{gathered}
$$


918 On the diffusion equation with an integral condition

where $\delta \gamma_{j}=\left(\gamma_{j}-\gamma_{j-1}\right) / h$. Obviously, the obtained problems are exactly those treated in Section 4 with $U_{0}-\gamma_{0}$ and $f_{j}-\delta \gamma_{j}$ instead of $U_{0}$ and $f_{j}$, respectively. Therefore, recalling the function $\gamma$ defined in (2.14), they are seen to be the timediscretized problems corresponding to the following evolution problem:

$$
\begin{gathered}
\frac{\partial \tilde{u}}{\partial t}-\frac{\partial^{2} \tilde{u}}{\partial x^{2}}=\tilde{f} \quad \text { in }(0,1) \times I, \\
\tilde{u}(x, 0)=\tilde{U}_{0}(x), \quad x \in(0,1), \\
\frac{\partial \tilde{u}}{\partial x}(0, t)=0, \quad t \in I, \\
\int_{0}^{1} \tilde{u}(x, t) d x=0, \quad t \in I,
\end{gathered}
$$

where

$$
\tilde{f}=f-\frac{d \gamma}{d t}, \quad \tilde{U}_{0}=U_{0}-\gamma_{0}
$$

It is easy to check, in virtue of assumption $\left(\mathrm{A}_{2}\right)$, that $d \gamma / d t \in C^{0,1}\left(I, B_{2}^{1}(0,1)\right)$. Hence, the same holds for $\tilde{f}$ thanks to assumption $\left(\mathrm{A}_{1}\right)$. Furthermore, owing to assumptions $\left(\mathrm{A}_{3}\right)$ and $\left(\mathrm{A}_{4}\right)$, it follows that $\tilde{U}_{0} \in H^{2}(0,1), \tilde{U}_{0}^{\prime}(0)=0$, and $\int_{0}^{1} \tilde{U}_{0}(x) d x=0$. Thus, since all assumptions of the preceding section are fulfilled, Theorem 4.8 is valid for problem (5.5)-(5.8). This fact allows us to state our main result

Theorem 5.1. Under assumptions $\left(A_{1}\right),\left(A_{2}\right),\left(A_{3}\right)$, and $\left(A_{4}\right)$, problem (2.2)-(2.5) admits a unique weak solution $u$ in the sense of Definition 2.1 having the following additional properties:

(i) $u \in C^{0,1}\left(I, B_{2}^{1}(0,1)\right) \cap L^{\infty}\left(I, L^{2}(0,1)\right)$ and $d u / d t \in L^{\infty}\left(I, B_{2}^{1}(0,1)\right)$;

(ii) the sequence of Rothe functions (3.15) converges to $u$ in the following sense:

$$
\begin{gathered}
u^{(n)} \rightarrow u \quad \text { in } L^{2}\left(I, L^{2}(0,1)\right), \\
u^{(n)} \rightarrow u \quad \text { in } C\left(I, B_{2}^{1}(0,1)\right), \\
\frac{d u^{(n)}}{d t} \rightarrow \frac{d u}{d t} \quad \text { in } L^{2}\left(I, B_{2}^{1}(0,1)\right),
\end{gathered}
$$

as $n \rightarrow \infty$;

(iii) $u$ depends continuously on the right-hand side of (2.2) and on the initial and boundary data; namely, a constant $K>0$ exists such that

$$
\|u\|_{C\left(I, B_{2}^{1}(0,1)\right)} \leqslant K\left(\|f\|_{C\left(I, B_{2}^{1}(0,1)\right)}+\left\|U_{0}\right\|_{H^{2}(0,1)}+\|\alpha\|_{C^{1}(I, \mathbb{R})}+\|E\|_{C^{1}(I, \mathbb{R})}\right) .
$$


Proof. Consider the function

$$
u=\tilde{u}+\gamma
$$

Since both $\tilde{u}$ and $\gamma$ belong to $L^{2}\left(I, L^{2}(0,1)\right) \cap C\left(I, B_{2}^{1}(0,1)\right)$, the same holds for $u$. Similarly, we infer that $d u / d t=d \tilde{u} / d t+d \gamma / d t$ is in $L^{2}\left(I, B_{2}^{1}(0,1)\right)$. Invoking the second relation in (5.9), that is, $\tilde{U}_{0}=U_{0}-\gamma_{0}$, we see that $u(0)=U_{0}$, and from (5.8), it follows that

$$
\int_{0}^{1} u(x, t) d x=\int_{0}^{1} \gamma(x, t) d x=\int_{0}^{1}\left[\alpha(t)\left(x-\frac{1}{2}\right)+E(t)\right] d x=E(t) .
$$

Moreover, performing the substitutions $\tilde{f}=f-d \gamma / d t$ and (5.12) in the integral identity (4.54) written for $\tilde{u}$, we get

$$
\begin{aligned}
\int_{I}\left(\frac{d u}{d t}(t),\right. & v(t))_{B_{2}^{1}(0,1)} d t+\int_{I}(u(t), v(t)) d t \\
= & \int_{I}(f(t), v(t))_{B_{2}^{1}(0,1)} d t+\int_{I}(\gamma(t), v(t)) d t
\end{aligned}
$$

for all $v \in L^{2}(I, V)$. Thus, $u$ defined in (5.12) turns out to be a weak solution of problem (2.2)-(2.5). Besides, since the weak solution of (5.5)-(5.8) is unique, so is the solution of (2.2)-(2.5).

Theorem 5.1(i) is an immediate consequence of the regularity properties of $\tilde{u}$ and the fact that $\gamma \in C^{0,1}\left(I, B_{2}^{1}(0,1)\right) \cap L^{\infty}\left(I, L^{2}(0,1)\right)$ which results from assumption $\left(\mathrm{A}_{2}\right)$.

Before checking the convergence properties in Theorem 5.1(ii), we remark that the Rothe sequences $\left\{u^{(n)}\right\}_{n}$ and $\left\{\tilde{u}^{(n)}\right\}_{n}$, corresponding to problems (2.2)(2.5) and (5.5)-(5.8), respectively, are connected by the formula

$$
u^{(n)}(t)=\tilde{u}^{(n)}(t)+\gamma^{(n)}(t) \quad \forall t \in I,
$$

where

$$
\gamma^{(n)}(t)=\gamma_{j-1}+\delta \gamma_{j}\left(t-t_{j-1}\right), \quad t \in\left[t_{j-1}, t_{j}\right], j=1, \ldots, n
$$

But, for all $t \in\left[t_{j-1}, t_{j}\right], j=1, \ldots, n$, we have

$$
\begin{aligned}
\left\|\gamma(t)-\gamma^{(n)}(t)\right\| & \leqslant|| \gamma(t)-\gamma\left(t_{j-1}\right)||+|| \delta \gamma_{j}||\left|t-t_{j-1}\right| \\
& \leqslant 2 C_{1}\left|t-t_{j-1}\right| \\
& \leqslant \frac{C}{n}
\end{aligned}
$$


where $C_{1}=(1 / \sqrt{12}) \sup _{t \in I}|(d \alpha / d t)(t)|+\sup _{t \in I}|(d E / d t)(t)|$, and $C=2 C_{1} T$. Therefore,

$$
\sup _{t \in I}\left\|\gamma(t)-\gamma^{(n)}(t)\right\| \leqslant \frac{C}{n} \longrightarrow 0 \quad \text { for } n \longrightarrow \infty
$$

that is, $\gamma^{(n)} \rightarrow \gamma$ in $C\left(I, L^{2}(0,1)\right) \subset C\left(I, B_{2}^{1}(0,1)\right)$ so that, according to the convergence properties of the sequence $\left\{\tilde{u}^{(n)}\right\}_{n}$, we conclude that $u$ is the weak limit in $L^{2}\left(I, L^{2}(0,1)\right)$ and the strong limit in $C\left(I, B_{2}^{1}(0,1)\right)$ to the Rothe sequence $\left\{u^{(n)}\right\}_{n}$. On the other hand, since

$$
\frac{d \gamma^{(n)}}{d t}(t)=\delta \gamma_{j}, \quad t \in\left(t_{j-1}, t_{j}\right], j=1, \ldots, n,
$$

it follows that $\left\|\left(d \gamma^{(n)} / d t\right)(t)\right\|_{B_{2}^{1}(0,1)} \leqslant C$ for all $t \in I$, which implies that the sequence $\left\{d \gamma^{(n)} / d t\right\}_{n}$ is bounded in $L^{2}\left(I, B_{2}^{1}(0,1)\right)$ and hence contains a subsequence $\left\{d \gamma^{\left(n_{k}\right)} / d t\right\}_{k}$ converging weakly to some function in $L^{2}\left(I, B_{2}^{1}(0,1)\right)$, which is shown to be equal to the function $d \gamma / d t$ in a similar way as in the proof of Theorem 4.3. Thus, since $d \tilde{u}^{(n)} / d t \rightarrow d \tilde{u} / d t$ in $L^{2}\left(I, B_{2}^{1}(0,1)\right)$, we get

$$
\frac{d u^{\left(n_{k}\right)}}{d t}=\frac{d \tilde{u}^{\left(n_{k}\right)}}{d t}+\frac{d \gamma^{\left(n_{k}\right)}}{d t}-\frac{d \tilde{u}}{d t}+\frac{d \gamma}{d t}=\frac{d u}{d t} \quad \text { in } L^{2}\left(I, B_{2}^{1}(0,1)\right) .
$$

However, from the uniqueness of the weak solution of problem (2.2)-(2.5), it follows in the usual way that the entire sequence $\left\{d u^{(n)} / d t\right\}_{n}$ converges weakly to $d u / d t$ in $L^{2}\left(I, B_{2}^{1}(0,1)\right)$.

As for Theorem 5.1(iii), using (5.9) and (5.12), inequality (4.70) written for $\tilde{u}$ yields

$$
\begin{aligned}
& \|u\|_{C\left(I, B_{2}^{1}(0,1)\right)} \\
& \leqslant C\left[\|f\|_{C\left(I, B_{2}^{1}(0,1)\right)}+\left\|\frac{d \gamma}{d t}\right\|_{C\left(I, L^{2}(0,1)\right)}+\left\|U_{0}\right\|_{H^{2}(0,1)}+\left\|\gamma_{0}\right\|_{H^{2}(0,1)}\right] \\
& \quad+\|\gamma\|_{C\left(I, L^{2}(0,1)\right) .}
\end{aligned}
$$

An easy computation leads to

$$
\begin{gathered}
\|\gamma(t)\|=\sqrt{\frac{1}{12}(\alpha(t))^{2}+(E(t))^{2}} \leqslant \frac{1}{\sqrt{12}}|\alpha(t)|+|E(t)|, \\
\|\gamma(t)\|_{H^{2}(0,1)}=\sqrt{\frac{13}{12}(\alpha(t))^{2}+(E(t))^{2}} \leqslant \sqrt{\frac{13}{12}}|\alpha(t)|+|E(t)|, \\
\left\|\frac{d \gamma}{d t}(t)\right\|=\sqrt{\frac{1}{12}\left(\frac{d \alpha}{d t}(t)\right)^{2}+\left(\frac{d E}{d t}(t)\right)^{2}} \leqslant \frac{1}{\sqrt{12}}\left|\frac{d \alpha}{d t}(t)\right|+\left|\frac{d E}{d t}(t)\right|,
\end{gathered}
$$


for every fixed $t \in I$, from which it follows that

$$
\begin{gathered}
\|\gamma\|_{C\left(I, L^{2}(0,1)\right)} \leqslant \frac{1}{\sqrt{12}}\|\alpha\|_{C(I, \mathbb{R})}+\|E\|_{C(I, \mathbb{R})}, \\
\|\gamma\|_{C\left(I, H^{2}(0,1)\right)} \leqslant \sqrt{\frac{13}{12}}\|\alpha\|_{C(I, \mathbb{R})}+\|E\|_{C(I, \mathbb{R})}, \\
\left\|\frac{d \gamma}{d t}\right\|_{C\left(I, L^{2}(0,1)\right)} \leqslant \frac{1}{\sqrt{12}}\left\|\frac{d \alpha}{d t}\right\|_{C(I, \mathbb{R})}+\left\|\frac{d E}{d t}\right\|_{C(I, \mathbb{R})}
\end{gathered}
$$

Accordingly, substituting in (5.21), we finally get estimation (5.11) with $K=$ $2 \max (C, 1)$. So we complete the proof.

\section{References}

[1] N.-E. Benouar and N. I. Yurchuk, A mixed problem with an integral condition for parabolic equations with a Bessel operator, Differentsial'nye Uravneniya 27 (1991), no. 12, 2094-2098 (Russian).

[2] A. Bouziani, Solution forte d'un problème mixte avec condition intégrale pour une classe d'équations paraboliques [Strong solutions of a mixed problem with an integral condition for a class of parabolic equations], Maghreb Math. Rev. 6 (1997), no. 1, 1-17 (French).

[3] On a class of parabolic equations with a nonlocal boundary condition, Acad. Roy. Belg. Bull. Cl. Sci. (6) 10 (1999), no. 1-6, 61-77.

[4] - On the solvability of parabolic and hyperbolic problems with a boundary integral condition, Int. J. Math. Math. Sci. 31 (2002), no. 4, 201-213.

[5] - On the weak solution of a three-point boundary value problem for a class of parabolic equations with energy specification, Abstr. Appl. Anal. 2003 (2003), no. $10,573-589$.

[6] J. R. Cannon, The solution of the heat equation subject to the specification of energy, Quart. Appl. Math. 21 (1963), 155-160.

[7] J. R. Cannon, Y. P. Lin, and A. L. Matheson, The solution of the diffusion equation in two space variables subject to the specification of mass, Appl. Anal. 50 (1993), no. 1-2, 1-15.

[8] J. R. Cannon, S. Pérez Esteva, and J. van der Hoek, A Galerkin procedure for the diffusion equation subject to the specification of mass, SIAM J. Numer. Anal. 24 (1987), no. 3, 499-515.

[9] J. R. Cannon and J. van der Hoek, The existence of and a continuous dependence result for the solution of the heat equation subject to the specification of energy, Boll. Un. Mat. Ital. Suppl. (1981), no. 1, 253-282.

[10] - An implicit finite difference scheme for the diffusion equation subject to the specification of mass in a portion of the domain, Numerical Solutions of Partial Differential Equations (Parkville, 1981) (J. Noye, ed.), North-Holland, Amsterdam, 1982, pp. 527-539.

[11] Diffusion subject to the specification of mass, J. Math. Anal. Appl. 115 (1986), no. 2, 517-529.

[12] M. Dehghan, Implicit locally one-dimensional methods for two-dimensional diffusion with a non-local boundary condition, Math. Comput. Simulation 49 (1999), no. 45, 331-349. 
[13] _ A finite difference method for a non-local boundary value problem for twodimensional heat equation, Appl. Math. Comput. 112 (2000), no. 1, 133-142.

[14] - Fully explicit finite-difference methods for two-dimensional diffusion with an integral condition, Nonlinear Anal., Ser. A: Theory Methods 48 (2002), no. 5, 637650.

[15] N. I. Ionkin, The solution of a certain boundary value problem of the theory of heat conduction with a nonclassical boundary condition, Differencial'nye Uravnenija 13 (1977), no. 2, 294-304 (Russian).

[16] J. Kačur, Method of Rothe in Evolution Equations, Teubner-Texte zur Mathematik, vol. 80, BSB B. G. Teubner Verlagsgesellschaft, Leipzig, 1985.

[17] J. Kačur and R. van Keer, On the numerical solution of semilinear parabolic problems in multicomponent structures with Volterra operators in the transmission conditions and in the boundary conditions, Z. Angew. Math. Mech. 75 (1995), no. 2, 91-103.

[18] L. I. Kamynin, A boundary value problem in the theory of heat conduction with nonclassical boundary conditions, Ž. Vyčisl. Mat. i Mat. Fiz. 4 (1964), no. 6, 1006-1024 (Russian).

[19] T. Nagasawa and S. Omata, Discrete Morse semiflows of a functional with free boundary, Adv. Math. Sci. Appl. 2 (1993), no. 1, 147-187.

[20] T. Nagasawa and A. Tachikawa, Existence and asymptotic behavior of weak solutions to strongly damped semilinear hyperbolic systems, Hokkaido Math. J. 24 (1995), no. 2, 387-405.

[21] V. Pluschke, Local solutions to quasilinear parabolic equations without growth restrictions, Z. Anal. Anwendungen 15 (1996), no. 2, 375-396.

[22] V. Pluschke and F. Weber, The local solution of a parabolic-elliptic equation with a nonlinear Neumann boundary condition, Comment. Math. Univ. Carolin. 40 (1999), no. 1, 13-38.

[23] K. Rektorys, The Method of Discretization in Time and Partial Differential Equations, Mathematics and Its Applications, vol. 4, D. Reidel Publishing, Dordrecht, 1982.

[24] E. Rothe, Zweidimensionale parabolische Randwertaufgaben als Grenzfall eindimensionaler Randwertaufgaben, Math. Ann. 102 (1930), 650-670 (German).

[25] M. P. Sapagovas and R. Yu. Chegis, On some boundary value problems with a nonlocal condition, Differential Equations 23 (1987), no. 7, 858-863, translation from Differentsial'nye Uravneniya 23 (1987), no. 7, 1268-1274.

[26] P. Shi, Weak solution to an evolution problem with a nonlocal constraint, SIAM J. Math. Anal. 24 (1993), no. 1, 46-58.

[27] N. I. Yurchuk, A mixed problem with an integral condition for some parabolic equations, Differentsial'nye Uravneniya 22 (1986), no. 12, 2117-2126 (Russian).

Nabil Merazga: Département de Mathématiques, Centre Universitaire Larbi Ben M’hidi, Oum El Bouagui 04000, Algeria

E-mail address: nabil.merazga@caramail.com

Abdelfatah Bouziani: Département de Mathématiques, Centre Universitaire Larbi Ben M’hidi, Oum El Bouagui 04000, Algeria

E-mail address: af_bouziani@hotmail.com 


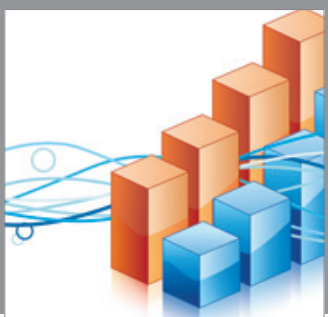

Advances in

Operations Research

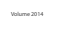

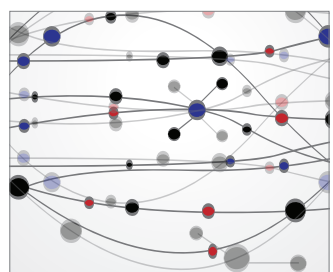

\section{The Scientific} World Journal
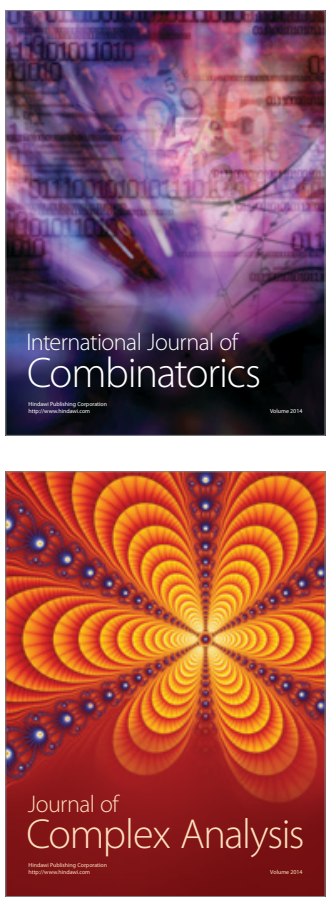

International Journal of

Mathematics and

Mathematical

Sciences
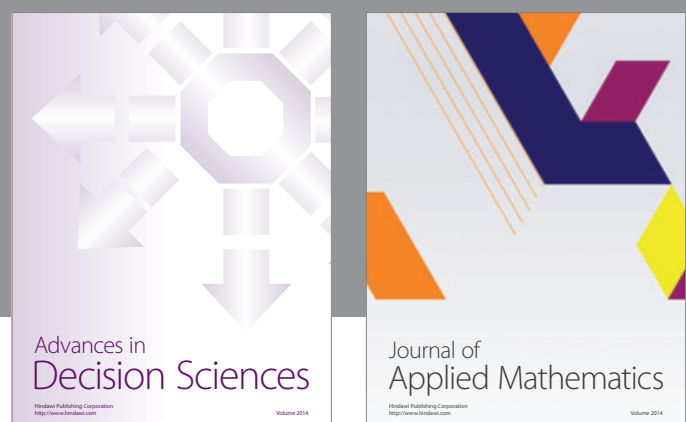

Journal of

Applied Mathematics
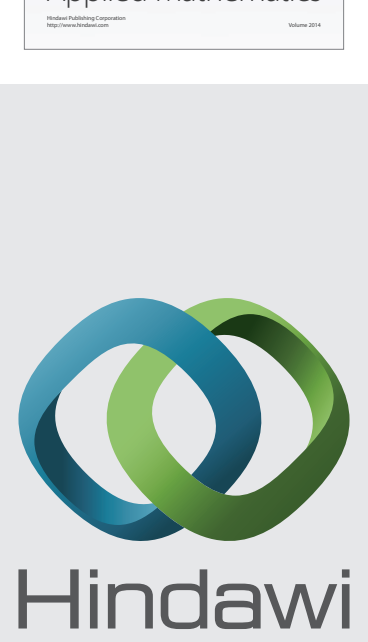

Submit your manuscripts at http://www.hindawi.com
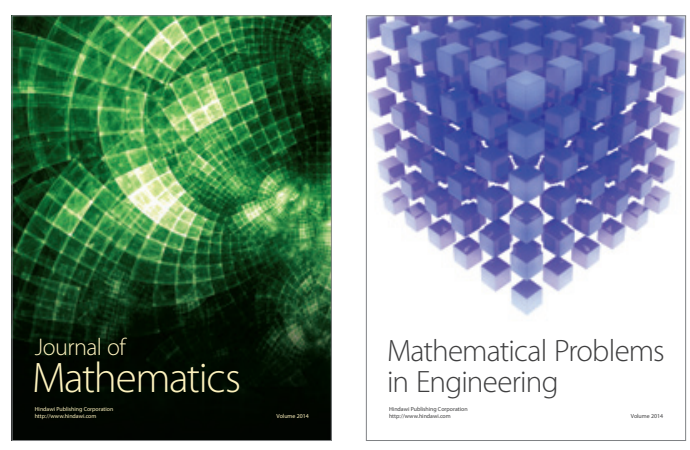

Mathematical Problems in Engineering
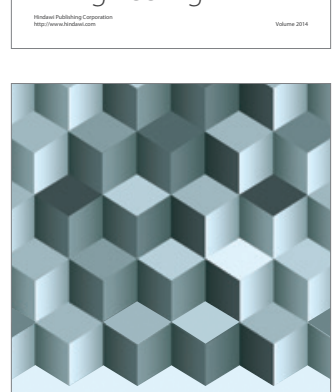

Journal of

Function Spaces
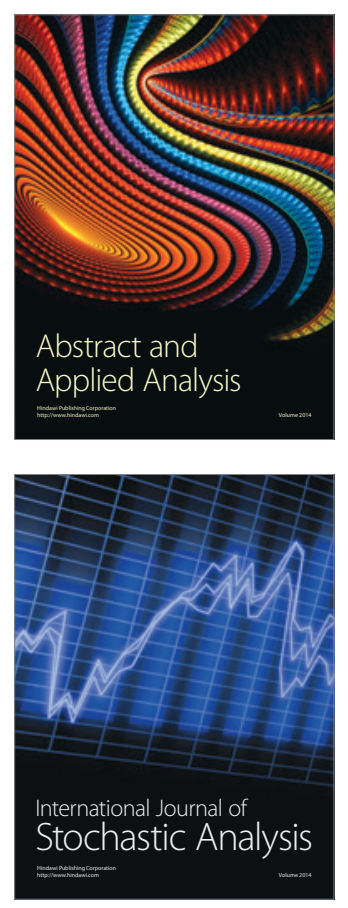

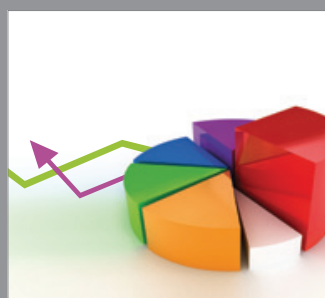

ournal of

Probability and Statistics

Promensencen
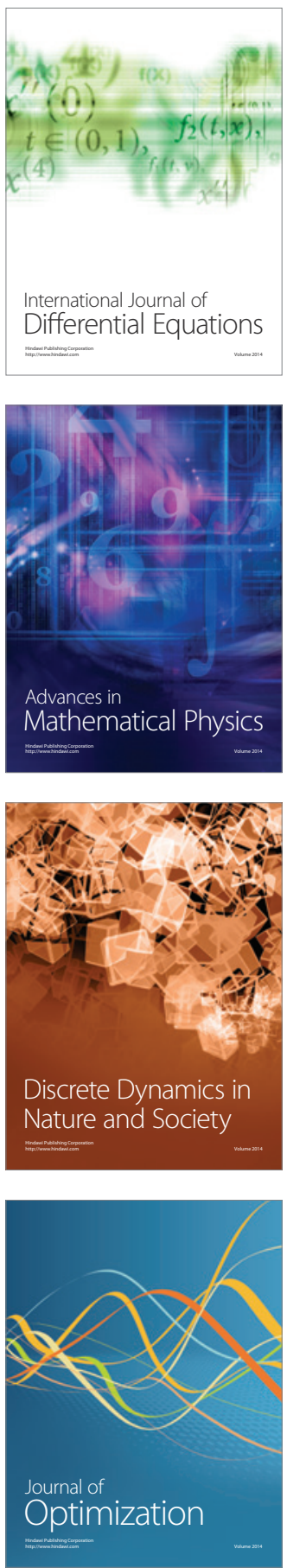